\title{
Phonon-induced dephasing of singlet-triplet superpositions in double quantum dots without spin-orbit coupling
}

\author{
K. Roszak ${ }^{1,2, \text { * }}$ and P. Machnikowski1, \\ ${ }^{1}$ Institute of Physics, Wroctaw University of Technology, 50-370 Wroctaw, Poland \\ ${ }^{2}$ Department of Condensed Matter Physics, Faculty of Mathematics and Physics, \\ Charles University, 12116 Prague, Czech Republic
}

\begin{abstract}
We show that singlet-triplet superpositions of two-electron spin states in a double quantum dot undergo a phonon-induced pure dephasing which relies only on the tunnel coupling between the dots and on the Pauli exclusion principle. As such, this dephasing process is independent of spin-orbit coupling or hyperfine interactions. The physical mechanism behind the dephasing is elastic phonon scattering, which persists to much lower temperatures than real phonon-induced transitions. Quantitative calculations performed for a lateral GaAs/AlGaAs gate-defined double quantum dot yield micro-second dephasing times at sub-Kelvin temperatures, which is consistent with experimental observations.

PACS numbers: 73.21.La, 03.65.Yz, 72.10.Di, 03.67.Lx
\end{abstract}

\section{INTRODUCTION}

The idea of encoding quantum information in spin states of electrons confined in quantum dots (QDs) is considered to be one of the most promising approaches to solid-state quantum computing. The original proposal of using single spins as quantum bits $\frac{1}{1}$ was followed by more sophisticated concepts in which a logical qubit was to be coded in a system of a few spins 2.3 . A class of proposed implementations uses two-spin states in double quantum dots (DQDs), assigning the quantum logical values to singlet and triplet spin configurations ${ }^{4}-\underline{\underline{6}}$. State-of-theart experimental techniques allow one to manipulate spin states of two electrons in a lateral, gate-defined DQD on time scales of hundreds of nanoseconds 7.8 , which has led to a renewed interest in factors that limit spin coherence.

From the point of view of coherent quantum applications, two kinds of decoherence are of interest. One is spin relaxation (thermalization of occupations) between the selected basis states (qubit states). The other one is dephasing of superpositions made of these states. Such a process, often referred to as pure dephasing, does not affect the occupations of the qubit basis states, thus conserving the classical information encoded in the system. However, by destroying quantum coherence, it degrades the quantum bit to a classical one and hence is detrimental for quantum information storage or processing. Moreover, if the system is initially prepared in a superposition of the basis states, the pure dephasing process will lead to a decay of this state. For a single electron (where the qubit states are usually associated with the Zeeman levels), this is equivalent to a decay of transverse components of the spin ${ }^{9}$.

Experimental results show that spin relaxation time between the Zeeman levels can be as long as milliseconds $\underline{10} \underline{-12}$. Similar values are obtained for relaxation of singlet-triplet superpositions in two-electron systems in double dots13,14. The triplet-singlet relaxation rate rapidly decreases already when the magnetic field reaches $0.1 \mathrm{~T}^{\underline{13}}$. Experimentally observed spin decoherence can be accounted for by theoretical models based on hyperfine coupling to the magnetic moments of lattice ions $15-20$. At higher fields, when the electron Zeeman splitting becomes much larger than the nuclear one, also phonon-assisted processes become important for spin relaxation. The latter may either result from an interplay of hyperfine and carrier-phonon interactions $\frac{15}{15}$ or are mediated by the spin-orbit coupling $21-26$. In a nearly degenerate singlet-triplet system, the hyperfine decoherence channel is predicted to persist even at strong magnetic fields 27 .

In strong contrast to these long relaxation times, the phase coherence observed in experiments decays many orders of magnitude faster. Time-averaged coherence times $\left(T_{2}^{*}\right)$ down to tens of nanoseconds have been observed ${ }^{8.28}$, while applying coherent control techniques yields an estimate of the intrinsic value on the order of a microsecond ${ }^{8}$.

In this paper, we show theoretically that an efficient pure dephasing channel is always present in systems of two electron spins localized in coupled semiconductor QDs. This channel results solely from the charge-phonon interaction in the presence of inter-dot tunnel coupling, and is essentially due to the distinguishability of singlet and triplet states via Pauli-blocking of certain transitions in the triplet case. The key feature of this decoherence process is that it does not require any spin-environment interaction and relies only on the mechanisms (tunnel coupling and the Pauli principle) that are essential for the implementation of quantum gates. In particular, it appears also in materials with negligible spin-orbit and hyperfine couplings and is essentially independent of the magnetic field.

The paper is organized as follows. In Sec. III we qualitatively explain the mechanism of dephasing. Next, in Sec. III we define the model to be studied and in Sec. IV we discuss its dynamics and derive the dephasing rates. The results are presented and discussed in Sec. V] Section VI concludes the paper. 


\section{COLLISIONAL DEPHASING MECHANISM}

Qualitatively, in the lowest energy state of the twoelectron system, each dot is occupied by a single electron. The spin configuration of the system may then be either singlet or triplet. In the former case, the orbital (spatial) wave function is symmetric and a transition to a doubly occupied state is possible ${ }^{29}$. This is forbidden by Pauli exclusion in the triplet configuration with an anti-symmetric orbital part. Such transitions are inefficient at sub-Kelvin temperatures because the doubly occupied state has a higher energy and the occupation of the required phonon states is negligible. However, a two-phonon process is still possible, in which the absorption of a phonon is followed by the re-emission of another one ${ }^{30,31}$. In such a process, the doubly occupied state is involved only "virtually" and energy conservation requires only that the two phonons have the same energy (that is, the scattering is elastic) but this energy can be arbitrary. Therefore, even at low temperatures, phonons scatter on a DQD in the singlet state, while a DQD in the triplet state is transparent to phonons. In this way, the singlet and triplet states can be distinguished by the macroscopic environment and each scattering event builds up correlation between the spin system and the crystal lattice. This distinguishability leads to pure dephasing of any singlet-triplet superposition, in some sense analogous to the "collisional decoherence" of the orbital degrees of freedom ${ }^{32,33}$. Although the intensity of this process drops down at low temperatures because of decreasing two-phonon spectral density at low frequencies, the temperature dependence is only polynomial (as opposed to exponential suppression of real transitions). As we will show, at sub-Kelvin temperatures, at which spin coherent control experiments on DQDs are performed, the two-phonon process can still lead to pure dephasing times as low as tens or hundreds of microseconds.

As the discussed process is phonon-assisted it is characterized by a large degree of irreversibility. This follows from the dispersive nature of the phonon reservoir which results in correlations with a macroscopic number of uncontrollable lattice degrees of freedom built up over the typical memory time of a phonon bath, which is on the order of a few picoseconds.

\section{MODEL}

In order to quantitatively estimate the effect of the dephasing process discussed above we consider two electrons in laterally coupled quantum dots interacting with a phonon reservoir. The dots are considered identical and the model is restricted to the ground state in each dot. This simplifying assumption strictly holds only when the Coulomb interaction between two electrons in a single dot is smaller than the single-particle excitation energy. Otherwise, virtual transitions to excited states would pro- vide another scattering channel. However, such scattering takes place both in the singlet and triplet states and can only yield distinguishability information due to the small exchange-related energy shifts between the relevant states and to the slight difference between the carrier densities in the lowest singlet and triplet states. Thus, in general, the scattering via excited single-particle states might lead to quantitative corrections to our results but it should not be expected to dominate the contribution from the channel described in Sec. II

The Hamiltonian of the system is therefore

$$
H=H_{\mathrm{DQD}}+H_{\mathrm{ph}}+H_{\mathrm{int}} .
$$

The first term describes the electrons and has the form

$$
\begin{aligned}
H_{\mathrm{DQD}}= & -t_{1} \sum_{s}\left(a_{\mathrm{L} s}^{\dagger} a_{\mathrm{R} s}+\text { h.c. }\right) \\
& +\frac{1}{2} \sum_{s, s^{\prime}} \sum_{i, j, k, l} V_{i j k l} a_{i s}^{\dagger} a_{j s^{\prime}}^{\dagger} a_{k s^{\prime}} a_{l s},
\end{aligned}
$$

where $a_{i s}, a_{i s}^{\dagger}$ are the electron annihilation and creation operators with $i=\mathrm{L}, \mathrm{R}$ denoting the left and right dot, respectively, and $s=\uparrow, \downarrow$ labeling the spin orientation. The first term in Eq. (11) accounts for single-particle interdot tunneling. The second term describes the Coulomb interaction, with $V_{i j k l}=V_{j i l k}=V_{k l i j}=V_{l k j i}$ (the wave functions may be chosen such that the matrix elements are real). For identical QDs the Coulomb matrix elements are also invariant under the interchange of the dots, L $\leftrightarrow$ R. Among the Coulomb terms, $V_{\mathrm{LRRL}}=$ $V_{\mathrm{RLLR}} \equiv U_{1}$ and $V_{\mathrm{LLLL}}=V_{\mathrm{RRRR}} \equiv U_{2}$ are the energies of the singly- and doubly-charged configurations, $V_{\mathrm{LRLR}}=V_{\mathrm{RLRL}} \equiv E_{\mathrm{X}}$ are exchange energies, $V_{\mathrm{LLRR}}=$ $V_{\mathrm{RRLL}} \equiv t_{\mathrm{C} 2}$ is the coupling between the doubly-charged configurations, while $V_{\text {RLLL }}$ and equivalent terms account for the coupling between the singly- and doubly-charged configurations and will be denoted by $t_{\mathrm{C}}$.

The Hamiltonian of the phonon reservoir is given by

$$
H_{\mathrm{ph}}=\sum_{\boldsymbol{k}, \lambda} \hbar \omega_{\boldsymbol{k}, \lambda} b_{\boldsymbol{k}, \lambda}^{\dagger} b_{\boldsymbol{k}, \lambda}
$$

where $b_{\boldsymbol{k}, \lambda}, b_{\boldsymbol{k}, \lambda}^{\dagger}$ are phonon annihilation and creation operators for a phonon from a branch $\lambda$ with a wave vector $\boldsymbol{k}$ and $\hbar \omega_{\boldsymbol{k}, \lambda}$ are the corresponding energies.

Since we formulate the model in terms of states localized in individual dots the overlap of the corresponding single particle wave functions is negligible and the off-diagonal phonon couplings (transferring the electrons between the dots) vanish. The electron-phonon interaction is therefore described by

$$
H_{\mathrm{int}}=\sum_{s, i} \sum_{\boldsymbol{k}, \lambda} F_{i}^{(\lambda)}(\boldsymbol{k}) a_{i s}^{\dagger} a_{i s}\left(b_{\boldsymbol{k}, \lambda}+b_{-\boldsymbol{k}, \lambda}^{\dagger}\right),
$$

where

$$
F_{\mathrm{L} / \mathrm{R}}^{(\lambda)}(\boldsymbol{k})=F^{(\lambda)}(\boldsymbol{k}) e^{ \pm i k_{x} D / 2}
$$


are coupling constants and $D$ is the inter-dot distance. We include the deformation potential and piezoelectric couplings. The coupling constants for the longitudinal $(\lambda=1)$ and transverse $\left(\lambda=t_{1,2}\right)$ acoustic phonon branches are, respectively ${ }^{34.35}$,

$$
F^{(1)}(\boldsymbol{k})=\sqrt{\frac{\hbar}{2 \rho_{\mathrm{c}} v \omega_{\boldsymbol{k}, 1}}}\left[\sigma k-i \frac{d e}{\varepsilon_{0} \varepsilon_{\mathrm{s}}} M_{\mathrm{l}}(\hat{\boldsymbol{k}})\right] \mathcal{F}(\boldsymbol{k}),
$$

and

$$
F^{\left(\mathrm{t}_{1}, \mathrm{t}_{2}\right)}(\boldsymbol{k})=-i \sqrt{\frac{\hbar}{2 \rho_{\mathrm{c}} v \omega_{\boldsymbol{k}, \mathrm{t}}}} \frac{d e}{\varepsilon_{0} \varepsilon_{\mathrm{s}}} M_{\mathrm{t}_{1}, \mathrm{t}_{2}}(\hat{\boldsymbol{k}}) \mathcal{F}(\boldsymbol{k}),
$$

where $e$ denotes the electron charge, $\rho_{\mathrm{c}}$ is the crystal density, $v$ is the normalization volume for the phonon modes, $d$ is the piezoelectric constant, $\varepsilon_{0}$ is the vacuum permittivity, $\varepsilon_{\mathrm{s}}$ is the static relative dielectric constant and $\sigma$ is the deformation potential constant. The functions $M_{\lambda}$ depend on the orientation of the phonon wave vector ${ }^{34.35}$. For the zinc-blende structure they are given by

$$
M_{\lambda}(\hat{\boldsymbol{k}})=2\left[\hat{k}_{x} \hat{k}_{y}\left(\hat{e}_{\lambda, \boldsymbol{k}}\right)_{z}+\hat{k}_{y} \hat{k}_{z}\left(\hat{e}_{\lambda, \boldsymbol{k}}\right)_{x}+\hat{k}_{z} \hat{k}_{x}\left(\hat{e}_{\lambda, \boldsymbol{k}}\right)_{y}\right],
$$

where $\hat{\boldsymbol{k}}=\boldsymbol{k} / k$ and $\hat{\boldsymbol{e}}_{\lambda, \boldsymbol{k}}$ are unit polarization vectors. The form factors $\mathcal{F}(\boldsymbol{k})$ depend on the wave function geometry and are given by

$$
\mathcal{F}(\boldsymbol{k})=\int d^{3} \boldsymbol{r} \psi^{*}(\boldsymbol{r}) e^{i \boldsymbol{k} \cdot \boldsymbol{r}} \psi(\boldsymbol{r}),
$$

where $\psi(\boldsymbol{r})$ is the envelope wave function of an electron centered at $\boldsymbol{r}=0$.

We will use the basis composed of the three triplet states

$$
\begin{aligned}
& \left|(1,1) T_{s}\right\rangle=a_{\mathrm{Ls}}^{\dagger} a_{\mathrm{Rs}}^{\dagger}|0\rangle, \quad s=\uparrow, \downarrow, \\
& \left|(1,1) T_{0}\right\rangle=\frac{a_{\mathrm{L} \uparrow}^{\dagger} a_{\mathrm{R} \downarrow}^{\dagger}-a_{\mathrm{R} \uparrow}^{\dagger} a_{\mathrm{L} \downarrow}^{\dagger}}{\sqrt{2}}|0\rangle
\end{aligned}
$$

and the three singlet states

$$
\begin{aligned}
|( \pm) S\rangle & =\frac{|(2,0) S\rangle \pm|(0,2) S\rangle}{\sqrt{2}} \\
& =\frac{\left(a_{\mathrm{L} \uparrow}^{\dagger} a_{\mathrm{L} \downarrow}^{\dagger} \pm a_{\mathrm{R} \uparrow}^{\dagger} a_{\mathrm{R} \downarrow}^{\dagger}\right)}{\sqrt{2}}|0\rangle, \\
|(1,1) S\rangle & =\frac{a_{\mathrm{L} \uparrow}^{\dagger} a_{\mathrm{R} \downarrow}^{\dagger}+a_{\mathrm{R} \uparrow}^{\dagger} a_{\mathrm{L} \downarrow}^{\dagger}}{\sqrt{2}}|0\rangle .
\end{aligned}
$$

The triplet states and the $|(1,1) S\rangle$ singlet involve electrons occupying separate QDs and, therefore, have lower energies than the other two singlet states.

The eigenstates of the Hamiltonian $H_{\mathrm{DQD}}$ are the three triplets with the energy $U_{1}-E_{\mathrm{X}}$, the singlet $|(-) S\rangle$ with the energy $E_{(-) S}=U_{2}-t_{\mathrm{C} 2}$, and the two states

$$
\begin{aligned}
& \left|S_{+}\right\rangle=\frac{1}{\sqrt{1+\xi^{2}}}[|(+) S\rangle+\xi|(1,1) S\rangle], \\
& \left|S_{-}\right\rangle=\frac{1}{\sqrt{1+\xi^{2}}}[|(1,1) S\rangle-\xi|(+) S\rangle],
\end{aligned}
$$

where

$$
\xi=\frac{2 \sqrt{2} t}{U+\sqrt{U^{2}+8 t^{2}}}
$$

with the eigenenergies $E_{ \pm}=\bar{E} \pm \sqrt{U^{2}+8 t^{2}} / 2$. Here $\bar{E}=\left(U_{2}+t_{\mathrm{C} 2}+U_{1}+E_{\mathrm{X}}\right) / 2, U=U_{2}+t_{\mathrm{C} 2}-U_{1}-E_{\mathrm{X}}$, and $t=\sqrt{2}\left(t_{\mathrm{C}}-t_{1}\right)$. In the weak tunneling regime, $t \ll U$, one has $\xi \ll 1$ and $\left|S_{+}\right\rangle \approx|(+) S\rangle,\left|S_{-}\right\rangle \approx|(1,1) S\rangle$. The degenerate triplet states and the singlet state $\left|S_{-}\right\rangle$are the lowest energy states. In the following, phase decoherence of a superposition of the $\left|S_{-}\right\rangle$singlet state and one of the triplet states is investigated.

Since the electron-phonon interaction conserves spin, the singlet state $\left|S_{-}\right\rangle$is not coupled by phonon-assisted transitions to the triplet states. Calculation shows that $\left|S_{-}\right\rangle$is also decoupled from $\left|S_{+}\right\rangle$, so the only nonzero off-diagonal matrix element of $H_{\text {int }}$ involving $\left|S_{-}\right\rangle$is

$$
\begin{aligned}
& \left\langle S_{-}\left|H_{\mathrm{int}}\right|(-) S\right\rangle= \\
& -\frac{2 i \xi}{\sqrt{1+\xi^{2}}} \sum_{\boldsymbol{k}, \lambda} F^{(\lambda)}(\boldsymbol{k}) \sin \left(\frac{k_{x} D}{2}\right)\left(b_{\boldsymbol{k}, \lambda}+b_{-\boldsymbol{k}, \lambda}^{\dagger}\right) .
\end{aligned}
$$

For this coupling, the spectral density of the phonon reservoir (as defined, e.g., in ${ }^{35}$ ) takes the form

$$
\begin{aligned}
R(\omega)= & \frac{1}{\hbar^{2}} \frac{4 \xi^{2}}{1+\xi^{2}} \sum_{\boldsymbol{k}, \lambda}\left|F^{(\lambda)}(\boldsymbol{k})\right|^{2} \sin ^{2}\left(\frac{k_{x} D}{2}\right) \\
& \times\left[\left(n_{\boldsymbol{k}, \lambda}+1\right) \delta\left(\omega-\omega_{\boldsymbol{k}, \lambda}\right)+n_{\boldsymbol{k}} \delta\left(\omega+\omega_{\boldsymbol{k}, \lambda}\right)\right]
\end{aligned}
$$

where $n_{\boldsymbol{k}, \lambda}$ is the Bose distribution.

\section{SYSTEM DYNAMICS AND DEPHASING RATES}

The two electron system is described by the reduced density matrix $\rho_{\mathrm{DQD}}=\operatorname{Tr}_{\mathrm{R}} \rho$, where $\operatorname{Tr}_{\mathrm{R}}$ denotes the partial trace over the reservoir degrees of freedom and $\rho$ is the density matrix of the complete system. Its evolution can be described using the time-convolutionless (TCL) projection operator method ${ }^{36}$. For factorized initial conditions (pure initial state), the TCL master equation takes the form

$$
\frac{d}{d t}[\mathcal{P} \rho(t)]=\mathcal{K}(t) \mathcal{P} \rho(t),
$$

where the projection operator $\mathcal{P}$ is defined by

$$
\mathcal{P} \rho(t)=\operatorname{Tr}_{\mathrm{R}} \rho(t) \otimes \rho_{\mathrm{R}},
$$

$\mathcal{K}(t)$ is the TCL generator, and $\rho_{\mathrm{R}}$ is the density matrix of the phonon reservoir at the thermal equilibrium. Expanding the TCL generator up to the fourth order yields 36

$$
\mathcal{K}(t)=\sum_{n=1}^{4} \mathcal{K}_{n}(t)
$$


with $\mathcal{K}_{1}(t)=\mathcal{K}_{3}(t)=0$ and

$$
\begin{aligned}
& \mathcal{K}_{2}(t)=\int_{0}^{t} d t_{1} \mathcal{P} \mathcal{L}(t) \mathcal{L}\left(t_{1}\right) \mathcal{P} \\
& \mathcal{K}_{4}(t)= \\
& \int_{0}^{t} d t_{1} \int_{0}^{t_{1}} d t_{2} \int_{0}^{t_{2}} d t_{3}\left[\mathcal{P} \mathcal{L}(t) \mathcal{L}\left(t_{1}\right) \mathcal{L}\left(t_{2}\right) \mathcal{L}\left(t_{3}\right) \mathcal{P}\right. \\
& \quad-\mathcal{P} \mathcal{L}(t)\left(\mathcal{L}\left(t_{1}\right) \mathcal{P} \mathcal{L}\left(t_{2}\right) \mathcal{L}\left(t_{3}\right)+\mathcal{L}\left(t_{2}\right) \mathcal{P} \mathcal{L}\left(t_{1}\right) \mathcal{L}\left(t_{3}\right)\right. \\
& \left.\left.+\mathcal{L}\left(t_{3}\right) \mathcal{P} \mathcal{L}\left(t_{1}\right) \mathcal{L}\left(t_{2}\right)\right) \mathcal{P}\right]
\end{aligned}
$$

where $\mathcal{L}(t)$ is the Liouville operator,

$$
\mathcal{L}(t) \rho\left(t^{\prime}\right)=-\frac{i}{\hbar}\left[H_{\text {int }}(t), \rho\left(t^{\prime}\right)\right]
$$

The coherence between the low-energy singlet and any of the triplet states is stored in the off-diagonal elements of the DQD density matrix,

$$
r_{i}=\left\langle S_{-}\left|\rho_{\mathrm{DQD}}\right|(1,1) T_{i}\right\rangle, \quad i=\uparrow, \downarrow, 0 .
$$

Upon explicitly evaluating the generators in Eqs. (3a,b), the evolution equation for any of these singlet-triplet offdiagonal elements can be written in the form

$$
\frac{d r_{i}}{d t}=K(t) r_{i}
$$

Here, we are interested in the loss of coherence, that is, in the evolution of the modulus of $r_{i}$. This is given by

$$
\frac{d\left|r_{i}\right|}{d t}=\operatorname{Re} K(t)\left|r_{i}\right|
$$

The function $K(t)$ varies with time only for $t \lesssim \hbar /\left(k_{\mathrm{B}} T\right)$ (reservoir memory time), which is of the order of picoseconds. Since the time scales relevant for the present discussion are many orders of magnitude longer, we can safely use its long-time (Markovian) limit $K \equiv K(t=$ $+\infty)$. Then, $K$ may be separated into two parts $K=$ $K^{(1)}+K^{(2)}$. The first term is

$$
\operatorname{Re} K^{(1)}=-\pi(1-\epsilon) R\left(-\omega_{0}-\Delta E\right),
$$

where $\hbar \omega_{0}=E_{(-) S}-E_{-}$is the energy difference between the two relevant singlet states,

$$
\begin{aligned}
\epsilon= & \int_{-\infty}^{\infty} d \omega\left[\frac{R\left(\omega_{0}-\omega\right)+R\left(\omega_{0}+\omega\right)-2 R\left(\omega_{0}\right)}{2 \omega^{2}}\right. \\
& +\frac{R\left(\omega_{0}+\omega\right)+R\left(-\omega_{0}-\omega\right)}{4 \omega_{0} \omega} \\
& \left.-\frac{R\left(\omega_{0}-\omega\right)+R\left(-\omega_{0}+\omega\right)}{4 \omega_{0} \omega}\right], \\
\Delta E= & \int_{-\infty}^{\infty} d \omega\left[\frac{R\left(\omega_{0}+\omega\right)+R\left(-\omega_{0}-\omega\right)}{4 \omega_{0} \omega}\right. \\
& \left.-\frac{R\left(\omega_{0}-\omega\right)+R\left(-\omega_{0}+\omega\right)}{4 \omega_{0} \omega}\right],
\end{aligned}
$$

and the spectral density of the phonon reservoir $R(\omega)$ is given by Eq. (2). This term describes the Fermi golden

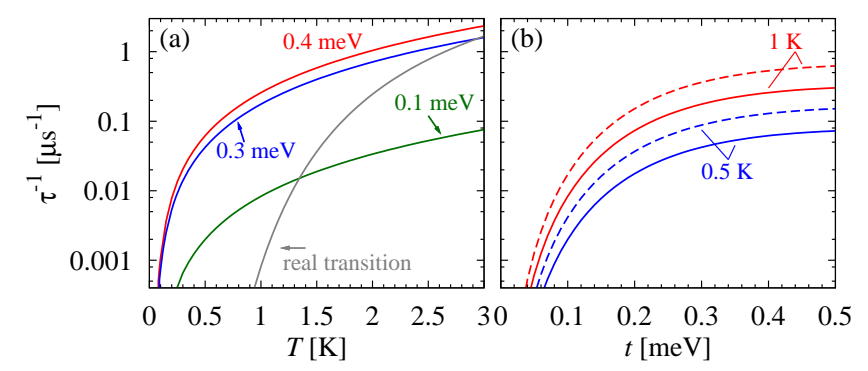

FIG. 1: Two-phonon induced pure dephasing rates: (a) As a function of temperature for different tunneling parameters. (b) As a function of the tunneling parameter for different temperatures. Gray line in (a) shows the dephasing rates from single-phonon transitions for $t=0.3 \mathrm{meV}$. All solid lines correspond to $D=200 \mathrm{~nm}$, while the dashed lines in (b) show results for $D=300 \mathrm{~nm}$.

rule rate of real single-phonon transitions between the $\left|S_{-}\right\rangle$and $|(-) S\rangle$ states with fourth order corrections due to phonon-induced energy shifts and coupling renormalizations. The second contribution is

$$
\begin{aligned}
& \operatorname{Re} K^{(2)}= \\
& \quad \frac{\pi}{2} \int_{-\infty}^{\infty} d \omega\left[\frac{2 R\left(\omega_{0}\right) R\left(-\omega_{0}\right)}{\omega^{2}}\right. \\
& \quad-\frac{R\left(\omega_{0}-\omega\right) R\left(-\omega_{0}+\omega\right)+R\left(\omega_{0}+\omega\right) R\left(-\omega_{0}-\omega\right)}{\omega^{2}} \\
& \left.\quad-\frac{R\left(\omega_{0}-\omega\right) R\left(-\omega_{0}+\omega\right)-R\left(\omega_{0}+\omega\right) R\left(-\omega_{0}-\omega\right)}{\omega_{0} \omega}\right]
\end{aligned}
$$

and accounts for the two-phonon elastic scattering process.

\section{RESULTS}

In the calculations, DQD geometry and material parameters are taken which correspond to lateral, gate-defined QDs made in the two-dimensional electron gas (2DEG) of a doped GaAs/AlGaAs interface heterostructure ${ }^{7.35}$. Two-dimensional Gaussian single electron wave functions are used with $170 \mathrm{~nm}$ full width at half maximum of the probability density. We set $U=$ $0.8 \mathrm{meV}$ and use the material parameters $c_{l}=5100 \mathrm{~m} / \mathrm{s}$, $c_{t}=2800 \mathrm{~m} / \mathrm{s}, \epsilon_{s}=13.2, d=0.16 \mathrm{C} / \mathrm{m}^{2}, \sigma=-0.8 \mathrm{eV}$, and $\rho_{c}=5360 \mathrm{~kg} / \mathrm{m}^{3}$. For simplicity, we use GaAs bulk phonon modes.

The pure dephasing rates resulting from the twophonon (scattering) process are shown in Fig. 1(a) as a function of temperature. Dephasing rates due to the single-phonon assisted transition for $t=0.3 \mathrm{meV}$ are shown in the same figure for comparison. At low temperatures, at which experiments on DQD ensembles are performed, the single-phonon transition is suppressed. At these temperatures, the dominating decoherence mechanism is the elastic scattering. This two-phonon process is much less influenced by decreasing the temperature 
since it involves only a virtual transition to a higher energy (doubly charged) singlet state. The resulting pure dephasing rates at sub-Kelvin temperatures are relatively high, compared to experimentally achievable gate operation times ${ }^{7}$.

Fig. 1(b) shows the pure dephasing rates as a function of the coupling parameter $t$. This parameter, which is also crucial for unitary operations on the two-qubit system, affects the dephasing rate via the energy difference between the singlet states, $\hbar \omega_{0}$, and via the mixing parameter $\xi$, which enters the spectral density in Eq. (2). Obviously, the dephasing vanishes for uncoupled dots. However, for non-zero coupling, the dephasing rate grows rapidly with $t$. One can see that the dephasing rate increases as the distance between the dots $D$ grows, which is a general feature of scattering-induced dephasing 32,33 .

\section{CONCLUSION}

We have shown that inter-dot tunneling and the Pauli principle, which are necessary for two-spin quantum gate operation in two-electron DQDs, lead to pure dephasing of singlet-triplet superpositions by elastic phonon scattering. Although this process does not affect occupations of the singlet and triplet states it irreversibly destroys the phase coherence of quantum states by correlating the spin system with a macroscopic number of lattice degrees of freedom which cannot be controlled in any way.

This dephasing process does not depend on any interactions between spins and their environment and is therefore qualitatively different from the dephasing mechanisms discussed so far. Unlike single-phonon-assisted real transitions between singlet states, which are suppressed at low temperatures, the two-phonon elastic process remains non-negligible in the sub-Kelvin range relevant for spin coherent control experiments on DQDs. For a gatedefined GaAs/InGaAs DQD system, this process leads to dephasing rate of the order of $10 \mu \mathrm{s}$ at $T=0.5 \mathrm{~K}$. This is consistent with the relatively short dephasing times found in experiments 7,8 . The elastic scattering mechanism does not depend on magnetic field, in contrast to processes mediated by the spin-orbit and hyperfine interactions.

\section{Acknowledgments}

This work was supported by the Polish MNiSW under Grant No. N N202 133633 and by the Czech Science Foundation under Grant No. 202/07/J051.
* Electronic address: katarzyna.roszak@pwr.wroc.pl

† Electronic address: pawel.machnikowski@pwr.wroc.pl

1 D. Loss and D. P. DiVincenzo, Phys. Rev. A 57, 120 (1998).

2 D. P. DiVincenzo, D. Bacon, J. Kempe, G. Burkard, and K. B. Whaley, Nature 408, 339 (2000).

3 P. Hawrylak and M. Korkusiński, Solid State Commun. 136, 508 (2005)

4 J. Levy, Phys. Rev. Lett. 89, 147902 (2002).

${ }^{5}$ M. S. Byrd and D. A. Lidar, Phys. Rev. Lett. 89, 047901 (2002).

${ }^{6}$ J. M. Taylor, H. A. Engel, W. Dür, A. Yacoby, C. M. Marcus, P. Zoller, and M. D. Lukin, Nature Physics 1, 177 (2005).

7 F. H. L. Koppens, C. Buizert, K. J. Tielrooij, I. T. Vink, K. C. Nowack, T. Meunier, L. P. Kouwenhoven, and L. M. K. Vandersypen, Nature 442, 766 (2006).

8 J. R. Petta, A. C. Johnson, J. M. Taylor, E. A. Laird, A. Yacoby, M. D. Lukin, C. M. Marcus, M. P. Hanson, and A. C. Gossard, Science 309, 2180 (2005).

9 I. A. Merkulov, A. L. Efros, and M. Rosen, Phys. Rev. B 65, 205309 (2002).

10 R. Hanson, B. Witkamp, L. M. K. Vandersypen, L. H. Willems van Beveren, J. M. Elzerman, and L. P. Kouvenhoven, Phys. Rev. Lett. 91, 196802 (2003).

11 J. M. Elzerman, R. Hanson, L. H. Willems van Beveren, B. Witkamp, L. M. K. Vandersypen, and L. P. Kouwenhoven, Nature 430, 431 (2004).

12 M. Kroutvar, Y. Ducommun, D. Heiss, M. Bichler, D. Schuh, G. Abstreiter, and J. J. Finley, Nature 432, 81 (2004).
13 A. C. Johnson, J. R. Petta, J. M. Taylor, A. Yacoby, M. D. Lukin, C. M. Marcus, M. P. Hanson, and A. C. Gossard, Nature 435, 925 (2005).

14 R. Hanson, L. H. Willems van Beveren, I. T. Vink, J. M. Elzerman, W. J. M. Naber, F. H. L. Koppens, L. P. Kouwenhoven, and L. M. K. Vandersypen, Phys. Rev. Lett. 94, 196802 (2005).

15 S. I. Erlingsson and Y. V. Nazarov, Phys. Rev. B 66, 155327 (2002).

16 G. Burkard, D. Loss, and D. P. DiVincenzo, Phys. Rev. B 59, 2070 (1999).

17 A. V. Khaetskii, D. Loss, and L. Glazman, Phys. Rev. Lett. 88, 186802 (2002).

18 J. M. Taylor, J. R. Petta, A. C. Johnson, A. Yacoby, C. M. Marcus, and M. D. Lukin, Phys. Rev. B 76, 035315 (2007).

19 J. Särkkä and A. Harju, Phys. Rev. B 79, 085313 (2009).

20 W. A. Coish and D. Loss, Phys. Rev. B 70, 195340 (2004).

21 V. N. Golovach, A. Khaetskii, and D. Loss, Phys. Rev. Lett. 93, 016601 (2004).

22 A. V. Khaetskii and Y. V. Nazarov, Phys. Rev. B 64, 125316 (2001).

23 J. I. Climente, A. Bertoni, G. Goldoni, M. Rontani, and E. Molinari, Phys. Rev. B 75, 081303(R) (2007).

24 J. I. Climente, A. Bertoni, G. Goldoni, M. Rontani, and E. Molinari, Phys. Rev. B 76, 085305 (2007).

${ }^{25}$ K. Shen and M. W. Wu, Phys. Rev. B 76, 235313 (2007).

26 V. N. Golovach, A. Khaetskii, and D. Loss, Phys. Rev. B 77, 045328 (2008).

27 W. A. Coish and D. Loss, Phys. Rev. B 72, 125337 (2005).

${ }^{28}$ F. H. L. Koppens, J. A. Folk, J. M. Elzerman, R. Hanson, L. H. Willems van Beveren, I. T. Vink, H. P. Tranitz, W. 
Wegscheider, L. P. Kouwenhoven, and L. M. K. Vandersypen, Science 309, 1346 (2005).

29 A. Grodecka, P. Machnikowski, and J. Förstner, Phys. Rev. B 78, 085302 (2008).

30 E. A. Muljarov and R. Zimmermann, Phys. Rev. Lett. 93, 237401 (2004).

31 E. A. Muljarov, T. Takagahara, and R. Zimmermann, Phys. Rev. Lett. 95, 177405 (2005).

${ }^{32}$ K. Hornberger and J. E. Sipe, Phys. Rev. A 68, 012105 (2003).
33 P. Machnikowski, Phys. Rev. Lett. 96, 140405 (2006).

34 G. D. Mahan, in Polarons in Ionic Crystals and Polar Semiconductors, edited by J. T. Devreese (North-Holland, Amsterdam, 1972).

35 K. Roszak, A. Grodecka, P. Machnikowski, and T. Kuhn, Phys. Rev. B 71, 195333 (2005).

${ }^{36}$ H.-P. Breuer and F. Petruccione, The Theory of Open Quantum Systems (Oxford University Press, Oxford, 2002). 